\title{
NGHIÊN CỬU ĐỘ CONG VÊNH CỦA SẢN PHẨM KHUNG NHỰA - KIM LOẠI TRONG ÉP PHUN DÙNG PHƯƠNG PHÁP TAGUCHI VÀ BỀ MẠT ĐÁP ÚNG
}

\author{
NGUYẼ̃N KHOA TRIỀ ${ }^{1}$, LEE BONG-KEE ${ }^{2}$ \\ ${ }^{1}$ Khoa Công nghệ Cơ Khí, Trường Đại học Công nghiệp thành phố Hồ Chí Minh \\ ${ }^{2}$ Khoa Co Khí, trường Đại học quốc gia Chonnam, Hàn Quốc \\ nguyenkhoatrieu@iuh.edu.vn
}

Tóm tắt. Trong bài này, một nghiên cứu mô phỏng số về độ cong vênh của một sản phẩm ép phun được thực hiện dựa trên phương pháp Taguchi và bề mặt đáp ứng. Để thu nhỏ và tích hợp các thành phần của bộ phận đèn nền cho màn hình tinh thể lỏng, một bộ khung tích hợp được phát triển bằng cách tích hợp khung thông thường, gương phản xạ kim loại và khung bezel. Dùng kỹ thuật ép phun chèn linh kiện, một sản phẩm kết hợp, mỏng nhưng độ cứng cao có thể được chế tạo thành công. Để tăng độ tin cậy cho sản xuất hàng loạt sản phẩm này, một nghiên cứu mô phỏng số về iỗi cong vênh đã được thực hiện. Ảnh hưởng của các thông số được phân tích bằng kỹ thuật tối ưu hóa kép. Theo đó, áp suất nén đóng vai trò quan trọng nhất; các giá trị tối ưu cho tất cả các thông số cũng được đề xuất. Nghiên cứu hiện tại có thể được sử dụng cho phát triển và sản xuất các sản phẩm mới với độ chính xác cao và tiêu thụ nguyên liệu tối thiểu.

Từ khóa. Ép phun, Cong vênh, Mô phỏng số, Taguchi, Phương pháp bề mặt đáp ứng, Tối ưu hóa kép.

\section{A STUDY OF WARPAGE FOR A NEWLY INSERT INJECTION-MOLDED FRAME USING TAGUCHI AND RESPONSE SURFACE METHOD}

\begin{abstract}
In the present study, a numerical investigation of warpage in injection-molded integrated mold frame units was carried out based on Taguchi method and Response Surface Methodology (RSM). In order to minimize and integrate the components of back light unit for a liquid crystal display, an integrated mold frame unit was developed by integrating conventional mold frame, metal reflector, and bezel together. Employing an insert injection molding, a slim composite part with a high strength could be successfully obtained. For a reliable mass production, it is required to minimize warpage defect by numerical investigation. The influences of parameters were analyzed based on Taguchi technique and RSM, as a result, packing pressure played the most significant role. Furthermore, the optimum conditions for all processing parameters were proposed. The present investigation can be used in a development and a reliable manufacturing of novel functional products with high precision and minimal material consumption.
\end{abstract}

Keywords. Insert injection molding, Warpage, Numerical analysis, Taguchi technique, Response surface methodology, Double optimization

\section{INTRODUCTION}

Ép phun chèn linh kiện (Insert Injection Molding - IIM) là một công nghệ sản xuất tiên tiến cho phép sản xuất hàng loạt các sản phẩm nhựa lai phức tạp. Bằng cách đặt chèn kim loại bên trong lòng khuôn trước khi điền đầy bằng polyme nóng chảy, một sản phẩm lai nhựa - kim loại có thể được sản xuất một cách chính xác. Nhìn chung, IIM có một số ưu điểm so với các phương pháp khác như chi phí thấp, chu kỳ ngắn, tính chất cơ học và chất lượng bề mặt của sản phẩm tốt. Do đó, nhiều sản phẩm lai nhựa - kim loại khác nhau, như vòng bi đúc, các bộ phận nẹp y tế, các bộ phận chip IC và các lõi ren cơ khí, đã được sản xuất bằng quy trình IIM [1-3].

Gần đây, chúng tôi đã sử dụng quy trình IIM để phát triển một đơn vị khung tích hợp mới để ứng dụng trong hệ thống đơn vị đèn nền (BLU) của màn hình tinh thể lỏng (LCD). Các thành phần tiêu biểu như khung khuôn, gương phản xạ kim loại và khung bezel được tích hợp, do đó tạo ra một bộ phận ghép nhựa - kim loại mỏng, nhẹ [4]. Mặc dù phần sản phẩm lai được thiết kế đã được sản xuất thành công bằng cách sử dụng quy trình IIM, việc tối ưu hóa các thông số cài đặt có liên quan là cần thiết để giảm cong vênh, cải thiện chất lượng sản phẩm.

Cong vênh được coi là một khiếm khuyết nghiêm trọng của ép phun thông thường. Để giải quyết hiệu ứng 
không mong muốn này, đã có nhiều nỗ lực nhằm nghiên cứu hiện tượng cong vênh của các sản phẩm ép phun bằng nhựa. Các tính chất của vật liệu cấu thành, hình dạng của sản phẩm, và các điều kiện cài đặt máy được coi là các yếu tố chính ảnh hưởng đến sự cong vênh. Trong số các yếu tố này, hầu hết các bài nghiên cứu tập trung vào các thông số cài đặt máy có liên quan và / hoặc tối ưu hóa của chúng để giảm thiểu cong vênh. Ví dụ, La và các cộng sự [5], Ozcelik và Sonat [6], Zheng và các cộng sự [7], Wang và các cộng sự [8], Azaman và các cộng sự [9], Zafosnik và các cộng sự [10] và Chen và các cộng sự [11] sử dụng phương pháp Taguchi để giảm thiểu sự cong vênh của các sản phẩm ép phun. Tương tự, Kurtaran và Erzurumlu [12], $\mathrm{Li}$ và các cộng sự [13] và Park và Dang [14], và sử dụng phương pháp bề mặt đáp ứng (RSM) để tối ưu hóa các thông số ảnh hưởng đến độ cong vênh. Chen và các cộng sự [15] sử dụng một kỹ thuật kép kết hợp của $R S M$ và một phần mềm thương mại lập trình phi tuyến (Lingo $\left.{ }^{\circledR}\right)$ để cải thiện kết quả từ phương pháp tối ưu hóa Taguchi. Nian và các cộng sự [16] sử dụng lý thuyết đường trung hòa để làm điều tương tự. Chen và Kurniawan [17] sử dụng sự kết hợp phương pháp Taguchi, một thuật toán di truyền (genetic algorithm), một mạng nơ-ron truyền ngược (back-propagation neural network - BPNN) để xác định điều kiện tối ưu. Hơn nữa, Xu và Yang [18] đề xuất một phương pháp tích hợp để cải thiện quá trình tối ưu hóa của họ, đó là sự kết hợp của phương pháp Taguchi, BPNN, phân tích tương quan xám (grey correlation analysis) và tối ưu hóa bầy đàn đa mục tiêu (multi objective particle swarm optimization).

Như đã mô tả, phương pháp Taguchi và RSM là các kỹ thuật thường được sử dụng để xác định các giá trị tối ưu của các thông số cài đặt có liên quan. Tuy nhiên, việc áp dụng một trong hai kỹ thuật này không cung cấp tối ưu hóa chính xác vì thuộc tính rời rạc của phương pháp Taguchi cũng như bản chất phức tạp của quá trình IIM. Hơn nữa, việc kết hợp một cấu trúc kim loại vào vật liệu polyme làm phức tạp việc tối ưu hóa quá trình IIM và cần phải xem xét các thông số bổ sung, cụ thể là nhiệt độ, cũng như các tính chất của kim loại và các thông số cài đặt có liên quan.

Để thực hiện điều này, một quy trình tối ưu hóa kép mới kết hợp cả phương pháp Taguchi và RSM được đề xuất trong nghiên cứu này. Phương pháp Taguchi được áp dụng với việc xem xét các thông số xử lý quan trọng của quy trình IIM, dẫn đến các giá trị tối ưu trung gian. Sau đó, các giá trị thu được này được sử dụng cho quá trình xử lý RSM tiếp theo để xác định các giá trị tối ưu được tinh chỉnh. Quá trình tối ưu hóa kép đã được áp dụng cho quy trình IIM để giảm thiểu sự cong vênh của khung khuôn tích hợp mới được phát triển. Với phương pháp này, các tác động của các thông số cài đặt đối với sự cong vênh của phần sản phẩm lai nhựa - kim loại đã được nghiên cứu sử dụng những điều kiện mang lại sự cong vênh tối thiểu.

\section{MÔ TẢ SẢN PHẨM LAI NHỤ๋A-KIM LOẠI}

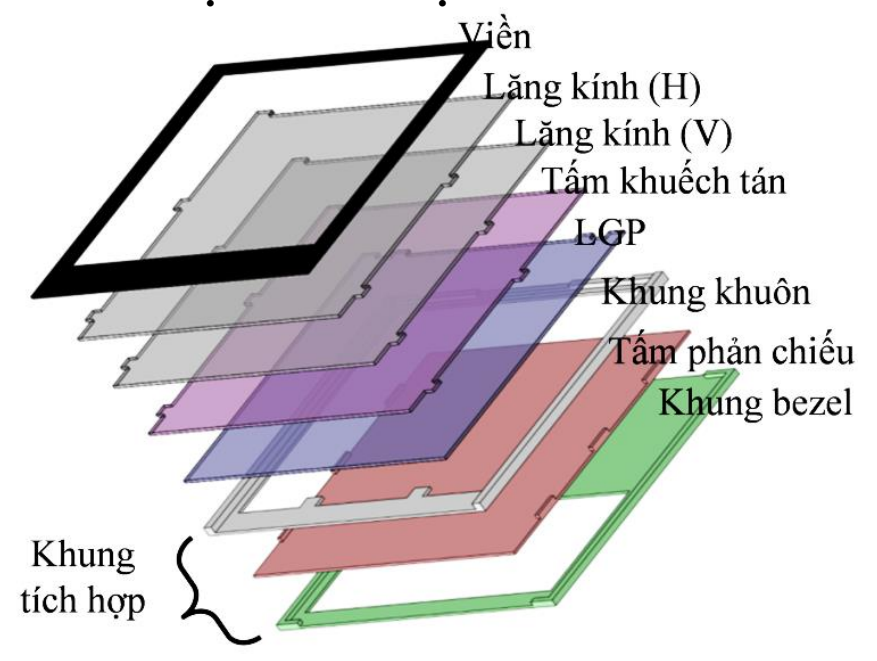

Hình 1: BLU tích hợp được phát triển trong nghiên cứu này.

Trong bài này, hiện tượng cong vênh của khung tích hợp LCD-BLU, bao gồm một phần nhựa và tấm kim loại, đã được nghiên cứu. Thông thường, LCD-BLU bao gồm các bộ phim quang học, bảng điều khiển ánh sáng, khung khuôn và gương phản xạ như minh họa trong hình 1 . Khung khuôn, gương phản chiếu và khung bezel được kết hợp để xây dựng bộ khung tích hợp. Phần tích hợp được sản xuất một cách hiệu quả bởi quy trình IIM bằng cách sử dụng khuôn ép như trong hình 2 . Hình $2 \mathrm{a}$ cho thấy lõi khuôn nằm trong phần khuôn di động của khuôn ép. Nó được thiết kế để có bốn lòng khuôn với bộ phản xạ kim loại được 
đưa vào trước khi đóng khuôn. Với khuôn này, sản phẩm bộ khung khuôn tích hợp lai nhựa - kim loại có thể được sản xuất thử. Hình $2 \mathrm{~b}$ cho thấy bốn sản phẩm ép được sản xuất ra bởi quy trình IIM.
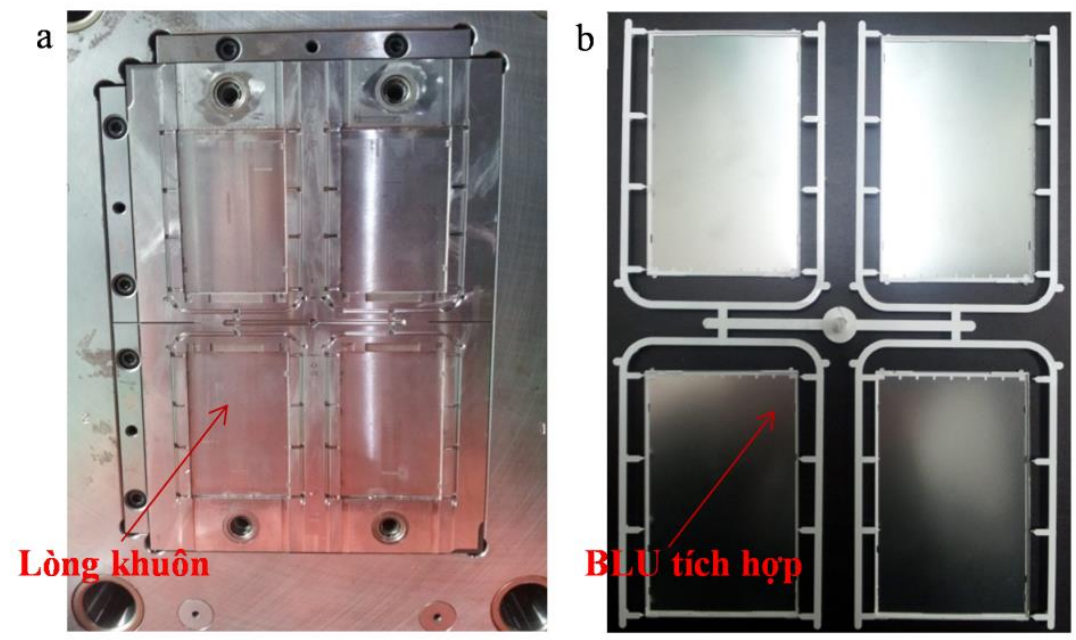

Hình 2: Hình thực tế của (a) khuôn và (b) sản phẩm.

Các vật liệu được sử dụng cho các bộ phản xạ kim loại và các phần bằng nhựa là thép không gỉ (JIS SUS410) có độ dày $0,2 \mathrm{~mm}$ và polycarbonate (Starex LB1020, Cheil). Bảng 1 cho thấy các thuộc tính quan trọng của hai vật liệu này, được sử dụng trong phân tích - mô phỏng số.

Bảng 1: Tính chất vật liệu của thép không gỉ và polycarbonate được sử dụng trong phân tích số.

\begin{tabular}{|cccc|}
\hline \multicolumn{2}{|c|}{ Thép không gỉ (JIS SUS410) } & \multicolumn{2}{c|}{ Polycarbonate $($ Starex LB 1020, Cheil) } \\
\hline Tỷ trọng $\left(\mathrm{kg} / \mathrm{m}^{3}\right)$ & $7.75 \times 10^{3}$ & Tỷ trọng $\left(\mathrm{kg} / \mathrm{m}^{3}\right)$ & $1.27 \times 10^{3}$ \\
Môđun đàn hồi $(\mathrm{GPa})$ & 200 & Môđun đàn hồi $(\mathrm{GPa})$ & 2.28 \\
Hệ số Poisson & 0.3 & Môđun trượt $(\mathrm{GPa})$ & 0.805 \\
CLTE $(1 / \mathrm{K})$ & $9.9 \times 10^{-6}$ & Hệ số Poisson & 0.417 \\
& & CLTE $(1 / \mathrm{K})$ & $7.3 \times 10^{-5}$ \\
\hline
\end{tabular}

Đối với phân tích - mô phỏng số, mô hình hình học được xây dựng cho toàn bộ sản phẩm, bao gồm các bộ phận bằng nhựa, bộ phản xạ kim loại và hệ thống phân phối bao gồm bạc cuống phun, kênh dẫn và miệng phun. Hình $3 \mathrm{a}$ cho thấy toàn bộ mô hình xây dựng dựa trên sản phẩm ép thực. Vì bốn lòng khuôn được thiết kế đối xứng và được đặt vào khuôn phun, một phần tư của mô hình, được biểu diễn trong hình $3 \mathrm{~b}$, được sử dụng trong phân tích - mô phỏng số để giảm thời gian tính toán cần thiết. Cần lưu ý rằng điều kiện biên đối xứng được gán trên mặt phẳng đối xứng của bạc cuống phun và kênh dẫn chính.

Trong nghiên cứu này, các phần tử tứ diện bốn nút được sử dụng để phân lập miền tính toán. Phần sản phẩm được xây dựng với 241.157 phần tử và 246.206 nút trong khi 754,423 phần tử và 758,358 nút đã được sử dụng cho hệ thống phân phối nhựa.

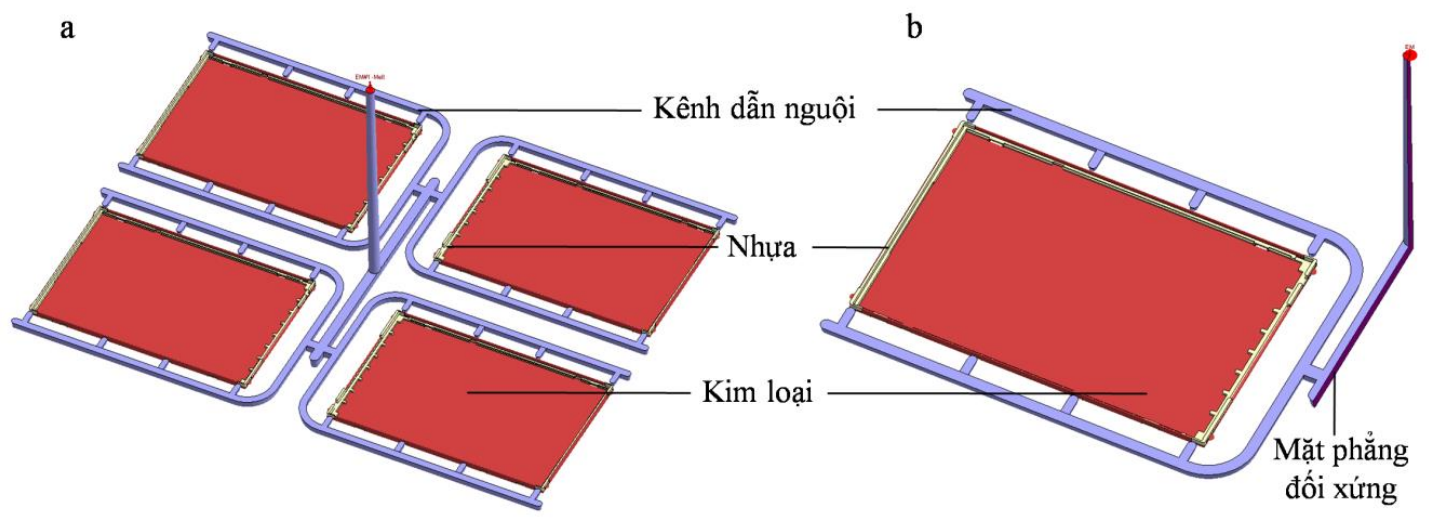

Hình 3: Mô hình hình học của khung khuôn tích hợp: (a) toàn bộ mô hình với 4 sản phẩm và (b) mô hình đối xứng cho phân tích số. 


\section{PHƯƠNG PHÁP}

\subsection{Mô phỏng sự cong vênh}

Trong nghiên cứu này, một phần mềm phân tích khuôn ép ba chiều (3D), Moldex3D ${ }^{\circledR}$, được sử dụng để phân tích toàn bộ quá trình IIM bao gồm các giai đoạn điền đầy, nén bảo áp và làm nguội. Trong quá trình éo nhựa, sự cong vênh của sản phẩm có nguồn gốc từ sự co ngót thể tích không cân bằng trong toàn bộ hình dạng hình học của phần nhựa khi nó nguội đi từ trạng thái nóng chảy đến trạng thái rắn. Sự biến thiên về nhiệt độ và áp suất gây ra những thay đổi về khối lượng và tỷ trọng của vật liệu polyme, do đó dẫn đến sự cong vênh của phần nhựa.

Để phân tích chính xác hiện tượng cong vênh, phần mềm phân tích sử dụng phương trình Williams-LandelFerry (WLF) để tạo ra một mô hình Maxwell đàn nhớt tuyến tính. Phương trình WLF được sử dụng như một biểu thức thực nghiệm cho hệ số chuyển dịch (shift factor), và thường được sử dụng để mô hình hóa sự phụ thuộc vào nhiệt độ của vật liệu polyme $[19,20]$. Phương trình WLF được hiển thị dưới đây:

$$
\log a_{T}=-\frac{c_{1}\left(T-T_{r e f}\right)}{c_{2}+T-T_{r e f}}
$$

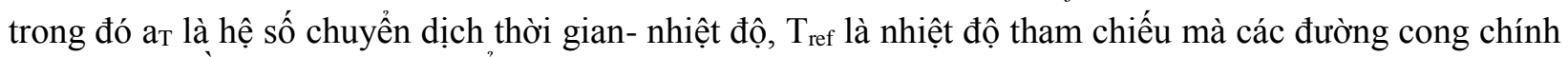
được tạo ra bằng cách dịch chuyển dữ liệu thử nghiệm động lực cơ học ở các nhiệt độ khác, và $\mathrm{C}_{1}$ và $\mathrm{C}_{2}$ là hệ số vật liệu được xác định bằng cách kết hợp dữ liệu thử nghiệm của hệ số dịch chuyển với phương trình WLF. Cần lưu ý rằng trong nghiên cứu này $17.4,51.6$ và $143^{\circ} \mathrm{C}$ được sử dụng tương ứng như là $\mathrm{C}_{1}, \mathrm{C}_{2}$ và $\mathrm{T}_{\text {ref. }}$

Mô hình Maxwell tổng quát được tạo ra bằng cách thêm nhiều phần tử Maxwell song song. Mô hình Maxwell tổng quát là một mô hình phổ biến được sử dụng để định lượng trạng thái giãn ra của vật liệu polyme. Sự giãn ra không xảy ra tại một thời điểm, mà thay vào đó trong một tập hợp thời gian do sự phân bố thời gian khác nhau do thực tế phân đoạn phân tử có chiều dài ngắn hơn đóng góp ít hơn phân đoạn phân tử có chiều dài dài hơn. Mô hình Maxwell tổng quát có thể được biểu diễn dưới dạng:

$$
\begin{aligned}
& E(t)=E_{\infty}+\sum_{i=1}^{n} E_{i} \exp \left(-\frac{t}{\lambda_{i}}\right) \\
& \lambda_{i} \equiv \frac{\eta_{i}}{E_{i}}
\end{aligned}
$$

Trong đó $\lambda_{\mathrm{i}}, \eta_{\mathrm{i}}$ và Ei là thời gian giãn ra, hệ số độ nhớt và mô đun đàn hồi của phần tử Maxwell thứ $\mathrm{i}, \mathrm{n}$ là tổng số phần tử Maxwell, và $\mathrm{E}_{\infty}$ là mô đun cuối cùng hoặc giá trị chuẩn cân bằng của mô đun đàn hồi. Hơn nữa, theo Fan và Kazmer [19], $\lambda_{\mathrm{i}}$ có thể được thay thế bằng $\mathrm{a}_{\mathrm{i}} \cdot \theta_{\mathrm{i}}$, trong đó $\theta_{\mathrm{i}}$ là thời gian thư giãn của phần tử Maxwell thứ $i$. Do đó, mô đun giãn tuyến tính của $\mathrm{E}(\mathrm{t}, \mathrm{T})$ có thể được biểu diễn một cách đơn giản thông qua các phương trình (1) và (2). Cần lưu ý rằng $\mathrm{E}_{\infty}$ được cài đặt giá trị $7.030 \mathrm{~Pa}$ và tám phần tử Maxwell được sử dụng.

Bảng 2: Các tham số cho mô hình Maxwell tổng quát.

\begin{tabular}{|cccccc|}
\hline Element & $\mathrm{E}_{\mathrm{i}}\left(\mathrm{dyne} / \mathrm{cm}^{2}\right)$ & $\lambda_{\mathrm{i}}(\mathrm{sec})$ & Element & $\mathrm{E}_{\mathrm{i}}\left(\mathrm{dyne} / \mathrm{cm}^{2}\right)$ & $\lambda_{\mathrm{i}}(\mathrm{sec})$ \\
\hline 1 & $1.16 \times 10^{9}$ & 0.002 & 5 & $2.89 \times 10^{6}$ & 10 \\
2 & $8.68 \times 10^{8}$ & 0.05 & 6 & $8.68 \times 10^{5}$ & 150 \\
3 & $1.16 \times 10^{8}$ & 0.35 & 7 & $3.61 \times 10^{5}$ & 3000 \\
4 & $1.45 \times 10^{7}$ & 1.5 & 8 & $1.74 \times 10^{5}$ & 40000 \\
\hline
\end{tabular}

Bảng 2 cho thấy các thông số vật liệu của mô hình Maxwell tổng quát, được cung cấp bởi Phòng thí nghiệm kiểm tra vật liệu Moldex3D, từ phép đo sử dụng áp kế Anton Paar MCR 502 và quy trình đo ASTM D4065 $\left(1\right.$ Pascal $=10$ dyne $\left./ \mathrm{cm}^{2}\right)$.

\subsection{Tối ưu hóa}

Tối ưu hóa trong quá trình IIM có thể được coi là một sự xác định các thông số cài đặt giảm thiểu hoặc tối đa hóa một thuộc tính đích, trong khi vẫn đáp ứng các ràng buộc. Trong trường hợp của nghiên cứu này, vấn đề tối ưu hóa là xác định các thông số cài đặt có liên quan đưa đến sự cong vênh tối thiểu của sản phẩm ép. Một vấn đề tối ưu hóa phi tuyến có ràng buộc điển hình đòi hỏi phải xác định một vector $\mathrm{x}$ là mức tối 
thiểu cục bộ cho hàm vô hướng $\mathrm{f}(\mathrm{x})$ tùy thuộc vào các ràng buộc trong các giá trị cho phép của $\mathrm{x}$; nói cách khác:

$$
\text { tìm min } f_{\vec{x}}(\vec{x}) \text { sao cho }\left\{\begin{array}{l}
c(\vec{x}) \leq 0 \\
c_{e q}(\vec{x})=0 \\
A \cdot \vec{x} \leq b \\
A_{e q} \cdot \vec{x}=b_{e q} \\
l b \leq \vec{x} \leq u b
\end{array}\right.
$$

Trong nghiên cứu này, một đoạn code MATLAB ${ }^{\circledR}$ ngắn được viết để xác định các giá trị tối ưu của các thông số cài đặt được xem xét. Vì các giá trị được tính toán khác nhau tùy thuộc vào điểm bắt đầu tối ưu hóa, toàn bộ dải thông số được chia thành một số phạm vi phụ với điểm bắt đầu được chỉ định cho mỗi điểm. Sử dụng cách tiếp cận này, có thể tránh được sự hội tụ vào mức tối ưu cục bộ, và thay vào đó, có thể đạt được mức tối ưu toàn thể trong phạm vi đang xét. Sau đó mô đun tối ưu hóa của Maple $₫$ được sử dụng để xác minh các giá trị tối ưu thu được bằng MATLAB®.

\subsection{Phương pháp bề mặt đáp ứng (RSM)}

RSM dùng để xác định mối quan hệ của các thông số và đáp ứng của chúng, được giới thiệu bởi $G$. E. $P$. Box và K. B. Wilson vào năm 1951, theo Bharti và Khan [21]. Họ đề xuất sử dụng mô hình đa thức bậc hai để thu được đáp ứng tối ưu từ các thí nghiệm. Mô hình này chỉ là một xấp xỉ, nhưng ước tính và áp dụng dễ dàng, ngay cả khi biết ít thông tin về quá trình. Mô hình bậc hai có dạng như sau:

$$
y=a_{0}+\sum_{i=1}^{k} a_{i} x_{i}+\sum_{i=1}^{k} a_{i i} x_{i}^{2}+\sum_{\substack{i, j=1 \\ i<j}}^{k} b_{i j} x_{i} x_{j}
$$

trong đó $\mathrm{x}_{\mathrm{i}}$ hoặc $\mathrm{x}_{\mathrm{j}}$ là các yếu tố, $\mathrm{a}_{\mathrm{i}}$ là các hệ số, $\mathrm{k}$ là số lượng các yếu tố.

Tổng số hệ số của mô hình trên được tính như sau:

$$
p=\frac{(k+1)(k+2)}{2}=36
$$

Mô hình toán học của đáp ứng mong muốn đối với một số yếu tố đầu vào độc lập được thực hiện bằng cách áp dụng phân tích hồi quy phi tuyến, sử dụng SPSS®, Design Expert® từ Stat-Ease,... hoặc tính tay bằng Excel® dùng phương pháp bình phương cực tiểu.

\subsection{Phương pháp tối ưu hóa kép}

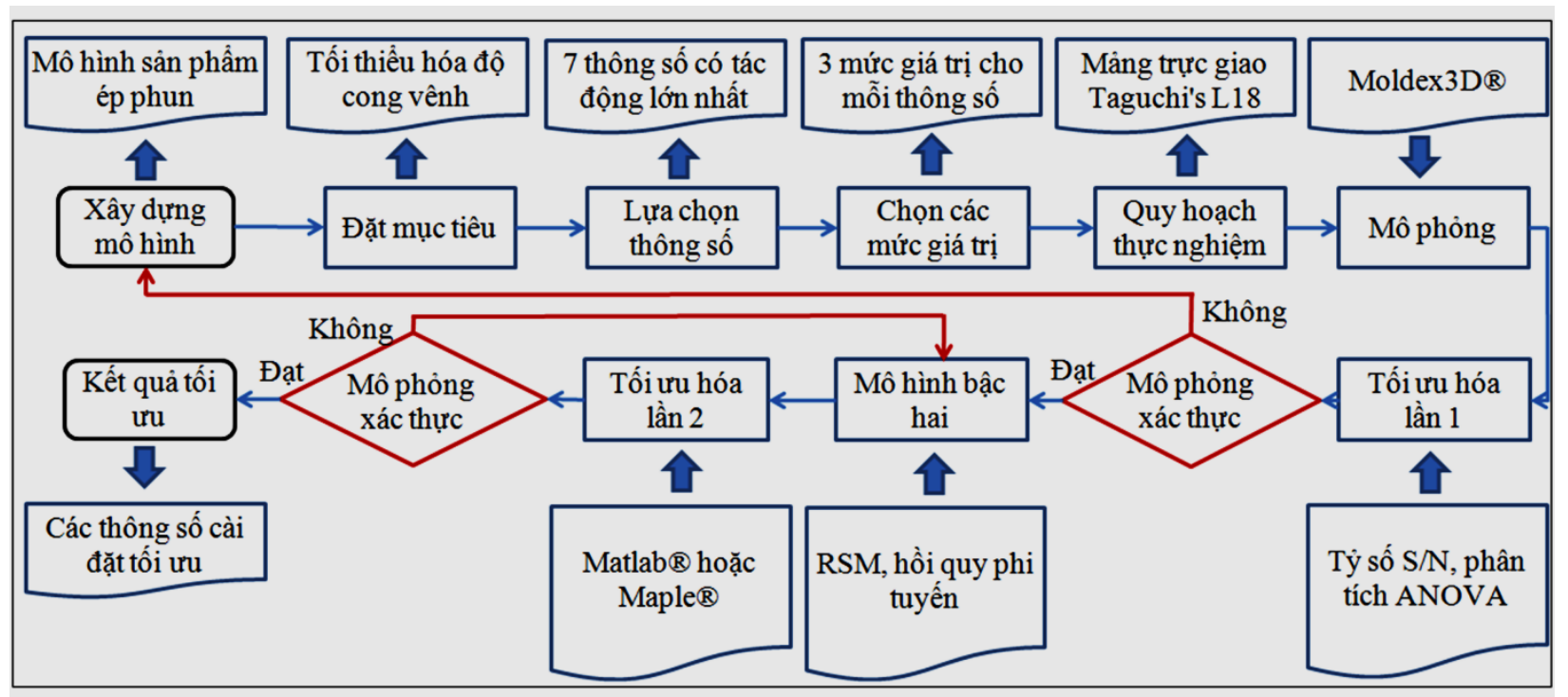

Hình 4: Lưu đồ quá trình tối ưu hóa kép.

Nhằm đạt được một quá trình IIM đáng tin cậy hơn, nghiên cứu này tập trung vào lỗi cong vênh, được coi là vấn đề quan trọng nhất trong các sản phẩm ép nhựa có thành mỏng hoặc trong bộ khung khuôn tích hợp, có tấm kim loại lớn hơn so với phần nhựa này. Sau khi xác định mục tiêu, chúng tôi liệt kê ra bảy thông số 
cài đặt quan trọng, bao gồm thời gian điền đầy, thời gian nén, áp suất nén, nhiệt độ nhựa nóng chảy, nhiệt độ khuôn, thời gian làm nguội và nhiệt độ ban đầu của phần kim loại chèn. Tiếp theo, phương pháp tối ưu hóa Taguchi được sử dụng để tối ưu hóa các thí nghiệm mô phỏng. Sau đó, kỹ thuật tối ưu hóa RSM được sử dụng để tìm ra giá trị tối ưu của mỗi thông số cài đặt (hình 4). Chúng tôi gọi đây là một quá trình tối ưu hóa kép, đã tận dụng cả ưu điểm của hai phương pháp Taguchi và RSM. Nó cho kết quả tốt hơn so với trường hợp chỉ sử dụng một trong hai phương pháp này một cách riêng biệt.

\section{KẾT QUẢ VÀ THẢO LUẬn}

\section{1 Ảnh hưởng của các thông số ép nhựa}

\section{a/ Lựa chọn thông số ép nhựa}

Có nhiều thông số có khả năng ảnh hưởng đến sự cong vênh của các sản phẩm lai nhựa-kim loại được sản xuất với quy trình IIM. Hầu hết các thông số cài đặt, như thời gian điền đầy, thời gian nén bảo áp, áp suất nén, nhiệt độ nóng chảy, nhiệt độ khuôn, thời gian làm nguội và nhiệt độ ban đầu của phần kim loại chèn, được xem xét đầu tiên để tối ưu hóa. Cần lưu ý rằng ảnh hưởng của hình dạng hình học của khuôn bao gồm bạc cuống phun, hệ thống kênh dẫn nhựa, và miệng phun được bỏ qua trong nghiên cứu này.

Như một sự tiếp nối của các nghiên cứu trước đó, nghiên cứu này tập trung vào việc tối ưu hóa các thông số cài đặt cho việc chạy máy. Thêm vào đó, ba mức của mỗi thông số cài đặt được thiết lập dựa trên các phạm vi được đề nghị. Bảng 3 liệt kê các thông số cài đặt và ba mức giá trị được sử dụng trong nghiên cứu này. Với cách tiếp cận này, một nghiên cứu dựa trên quy hoạch thực nghiệm (DOE) và mô hình bề mặt đáp ứng dựa trên đó có thể được xây dựng một cách hệ thống.

Bảng 3: Các thông số cài đặt và giá trị chi tiết của chúng trong nghiên cứu này.

\begin{tabular}{|c|l|c|c|c|}
\hline \multirow{2}{*}{ Ký hiệu } & \multicolumn{1}{|c|}{ Thông số } & \multicolumn{3}{|c|}{ Các mức giá trị } \\
\cline { 3 - 5 } & & 1 & 2 & 3 \\
\hline $\mathrm{A}$ & Thời gian điền đầy $(\mathrm{s})$ & 0.1 & 0.25 & 0.5 \\
\hline $\mathrm{B}$ & Thời gian nén $(\mathrm{s})$ & 1 & 3 & 5 \\
\hline $\mathrm{C}$ & Áp suất nén $(\mathrm{MPa})$ & 310 & 325 & 340 \\
\hline $\mathrm{D}$ & Nhiệt độ nhựa nóng chảy $\left({ }^{\circ} \mathrm{C}\right)$ & 290 & 300 & 310 \\
\hline $\mathrm{E}$ & Nhiệt độ khuôn $\left({ }^{\circ} \mathrm{C}\right)$ & 60 & 70 & 80 \\
\hline $\mathrm{F}$ & Thời gian làm nguội $(\mathrm{s})$ & 10 & 15 & 20 \\
\hline $\mathrm{G}$ & Nhiệt độ ban đầu của phần kim loại chèn $\left({ }^{\circ} \mathrm{C}\right)$ & 25 & 30 & 35 \\
\hline
\end{tabular}

Từ số các thông số được lựa chọn và các mức tương ứng của chúng, một tập hợp con của mảng trực giao $\mathrm{L}_{18}(21 \times 37)$ đã được chọn như trong bảng 4 . Các mô phỏng số của quá trình IIM cho sản phẩm lai được thực hiện bằng cách sử dụng các bộ tham số của mảng trực giao. Các mức độ cong vênh tối đa của sản phẩm, được dự đoán bởi các mô phỏng số, và tỷ số $\mathrm{S} / \mathrm{N}$ tương ứng cũng được liệt kê trong bảng 4 . Vì độ cong vênh, đặc điểm mục tiêu của nghiên cứu, cần phải được giảm thiểu để đạt được sản phẩm có chất lượng cao, định nghĩa $\mathrm{S} / \mathrm{N}$ "nhỏ hơn-tốt hơn" được sử dụng để tính toán tỷ số $\mathrm{S} / \mathrm{N}$.

Bảng 4: Mảng trực giao được sử dụng trong nghiên cứu này: Các thông chi tiết và độ cong vênh tối đa thu được bằng mô phỏng số (MSD là độ lệch chuẩn trung bình).

\begin{tabular}{|c|c|c|c|c|c|c|c|c|c|c|}
\hline \multirow{2}{*}{ STT } & \multicolumn{7}{|c|}{ Thông số } & \multirow{2}{*}{ Cong vênh } & \multirow{2}{*}{ MSD } & \multirow{2}{*}{ Tỷ số S/N } \\
\hline & A & B & $\mathrm{C}$ & $\mathrm{D}$ & $\mathrm{E}$ & $\mathrm{F}$ & G & & & \\
\hline 1 & 1 & 1 & 1 & 1 & 1 & 1 & 1 & 0.2086 & 0.043514 & 13.61371 \\
\hline 2 & 1 & 2 & 2 & 2 & 2 & 2 & 2 & 0.2008 & 0.040321 & 13.94473 \\
\hline 3 & 1 & 3 & 3 & 3 & 3 & 3 & 3 & 0.2332 & 0.054382 & 12.64543 \\
\hline 4 & 2 & 1 & 1 & 2 & 2 & 3 & 3 & 0.1854 & 0.034373 & 14.63781 \\
\hline 5 & 2 & 2 & 2 & 3 & 3 & 1 & 1 & 0.2147 & 0.046096 & 13.36336 \\
\hline 6 & 2 & 3 & 3 & 1 & 1 & 2 & 2 & 0.2172 & 0.047176 & 13.2628 \\
\hline 7 & 3 & 1 & 2 & 1 & 3 & 2 & 3 & 0.1910 & 0.036481 & 14.37933 \\
\hline 8 & 3 & 2 & 3 & 2 & 1 & 3 & 1 & 0.2172 & 0.047176 & 13.26280 \\
\hline 9 & 3 & 3 & 1 & 3 & 2 & 1 & 2 & 0.1907 & 0.036366 & 14.39299 \\
\hline 10 & 1 & 1 & 3 & 3 & 2 & 2 & 1 & 0.2235 & 0.049952 & 13.01445 \\
\hline 11 & 1 & 2 & 1 & 1 & 3 & 3 & 2 & 0.2011 & 0.040441 & 13.93176 \\
\hline 12 & 1 & 3 & 2 & 2 & 1 & 1 & 3 & 0.2053 & 0.042148 & 13.75222 \\
\hline 13 & 2 & 1 & 2 & 3 & 1 & 3 & 2 & 0.2109 & 0.044479 & 13.51847 \\
\hline
\end{tabular}



DƯNG PHƯƠNG PHÁP TAGUCHI VÀ BỀ MẠT ĐÁP ỨNG

\begin{tabular}{|l|lllllll|l|l|l|}
\hline 14 & 2 & 2 & 3 & 1 & 2 & 1 & 3 & 0.2160 & 0.046656 & 13.31092 \\
\hline 15 & 2 & 3 & 1 & 2 & 3 & 2 & 1 & 0.1916 & 0.036711 & 14.35209 \\
\hline 16 & 3 & 1 & 3 & 2 & 3 & 1 & 2 & 0.2094 & 0.043848 & 13.58047 \\
\hline 17 & 3 & 2 & 1 & 3 & 1 & 2 & 3 & 0.1918 & 0.036787 & 14.34303 \\
\hline 18 & 3 & 3 & 2 & 1 & 2 & 3 & 1 & 0.1990 & 0.039601 & 14.02294 \\
\hline \multicolumn{8}{|c|}{ Trû́ng } \\
\hline \multicolumn{8}{|c|}{ Trung bình }
\end{tabular}

\section{b/ Mức độ ảnh hưởng của các thông số}

Bảng 5 cho thấy kết quả tỷ số $\mathrm{S} / \mathrm{N}$ tóm tắt cho mỗi thông số cài đặt. Kết quả cũng được vẽ trong hình 5 , trong đó mô tả rõ ràng ảnh hưởng của các thông số cài đặt. Ví dụ, sự cong vênh của sản phẩm tăng khi thời gian điền đầy tăng (thông số $\mathrm{A}$ ), và khi áp lực nén giảm (thông số $\mathrm{C}$ ) trong phạm vi vận hành điển hình. Bằng cách đánh giá sự khác biệt giữa giá trị tỷ số $\mathrm{S} / \mathrm{N}$ tối đa và tối thiểu, ta có thể sự thấy được mức độ ảnh hưởng của mỗi thông số. Hình 6 cho thấy biểu đồ mức độ ảnh hưởng của các thông số cài đặt. Như thế, áp lực nén là thông số quan trọng nhất ảnh hưởng đến độ cong vênh.

Bảng 5: Kết quả tỷ số $\mathrm{S} / \mathrm{N}$ cho mỗi thông số cài đặt.

\begin{tabular}{|c|c|c|c|c|c|c|c|c|}
\hline Thông số & $\mathrm{A}$ & $\mathrm{B}$ & $\mathrm{C}$ & $\mathrm{D}$ & $\mathrm{E}$ & $\mathrm{F}$ & $\mathrm{G}$ & Tổng \\
\hline Mức 1 & 13.4837 & 13.7907 & 14.2119 & 13.7535 & 13.6255 & 13.6689 & 13.6048 & \multirow{2}{*}{41.2215} \\
\hline Mức 2 & 13.7409 & 13.6927 & 13.8301 & 13.9216 & 13.8873 & 13.8827 & 13.7718 \\
\hline Mức 3 & 13.9969 & 13.7380 & 13.1794 & 13.5462 & 13.7087 & 13.6698 & 13.8447 & \\
\hline Chênh lệch & 0.5132 & 0.0979 & 1.0324 & 0.3754 & 0.2618 & 0.2137 & 0.2399 & 2.7344 \\
\hline Ånh hưởng \% & 18.77 & 3.58 & 37.76 & 13.73 & 9.57 & 7.82 & 8.77 & 100 \\
\hline
\end{tabular}

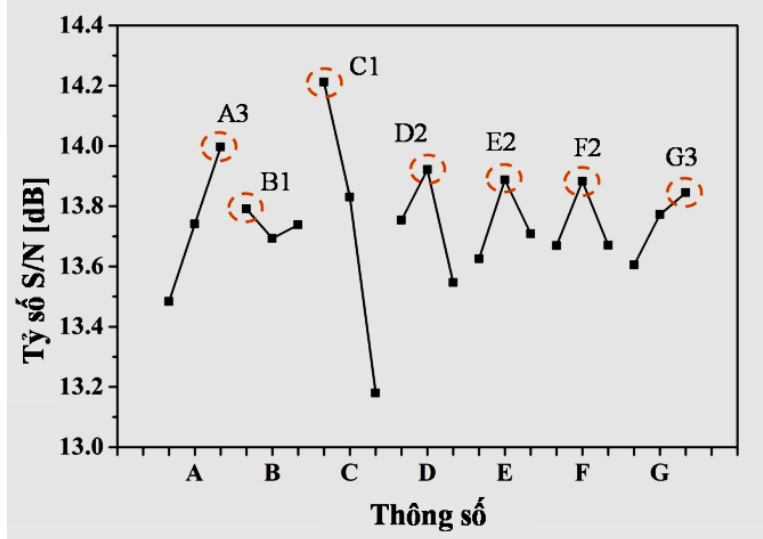

Hình 5: Kết quả phân tích tỷ số $\mathrm{S} / \mathrm{N}$ cho thấy ảnh hưởng của các thông số cài đặt đối với sự cong vênh.

Để tiếp tục nghiên cứu mức độ ảnh hưởng của các thông số cài đặt có liên quan, phương pháp ANOVA được thực hiện như trong bảng 6 . Phù hợp với phân tích tỷ số $\mathrm{S} / \mathrm{N}$, áp suất nén, thời gian điền đầy và nhiệt độ nóng chảy có ảnh hưởng lớn tới việc giảm độ cong vênh. Mặc dù có xu hướng tương tự giữa tỷ số $\mathrm{S} / \mathrm{N}$ và phân tích $A N O V A$, có sự khác biệt nhỏ về độ lớn của các thông số cài đặt. Phương pháp ANOVA chỉ cho thấy ba thông số gồm áp suất nén, thời gian điền đầy và nhiệt độ nóng chảy, vì có giá trị $\mathrm{F}$ lớn hơn

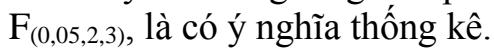

Bảng 6: Kết quả ANOVA.

\begin{tabular}{|cccccccc|}
\hline Thông số & $\mathrm{S}$ & $\mathrm{f}$ & $\mathrm{V}$ & $\mathrm{F}$ & $\mathrm{F}_{(0.05,2,3)}$ & $\mathrm{P} \%$ & Hạng \\
\hline $\mathrm{A}$ & 0.000449 & 2 & 0.0002245 & 24.575295 & 9.55209 & 15.44 & 2 \\
$\mathrm{~B}$ & $1.401 \times 10^{-5}$ & 2 & $7.007 \times 10^{-6}$ & 0.76706 & 9.55209 & 0.48 & \\
$\mathrm{C}$ & 0.0018578 & 2 & 0.0009289 & 101.69256 & 9.55209 & 63.89 & 1 \\
$\mathrm{D}$ & 0.0002551 & 2 & 0.0001276 & 13.963812 & 9.55209 & 8.77 & 3 \\
$\mathrm{E}$ & 0.0001124 & 2 & $5.619 \times 10^{-5}$ & 6.1510765 & 9.55209 & 3.86 & \\
$\mathrm{~F}$ & $9.937 \times 10^{-5}$ & 2 & $4.969 \times 10^{-5}$ & 5.4393018 & 9.55209 & 3.42 & 3.20 \\
G & $9.292 \times 10^{-5}$ & 2 & $4.646 \times 10^{-5}$ & 5.086425 & 9.55209 & & \\
\hline Sai số & $2.74 \times 10^{-5}$ & 3 & $9.134 \times 10^{-6}$ & & & & \\
Tổng & 0.002908 & 17 & & & & & \\
\hline
\end{tabular}




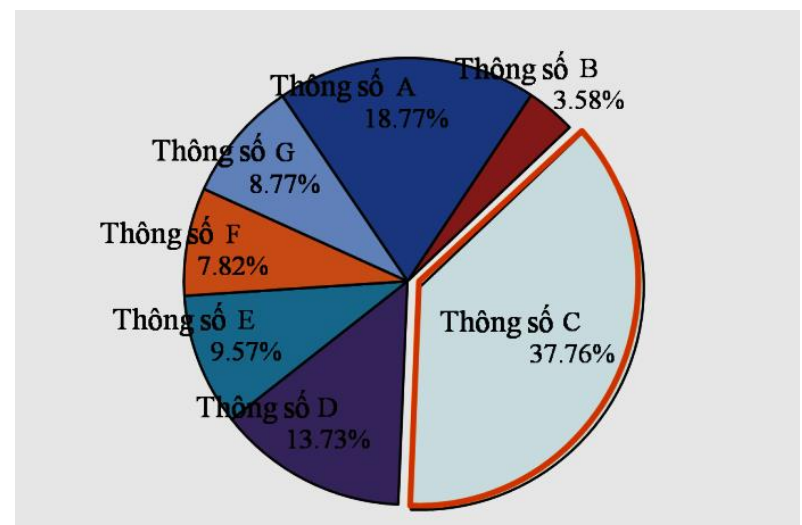

Hình 6: Mức độ ảnh hưởng của mỗi thông số cài đặt dựa trên phân tích tỷ số $\mathrm{S} / \mathrm{N}$.

Để phân tích sâu hơn tác động của ba thông số cài đặt quan trọng này, độ cong vênh tại bốn góc của sản phẩm lai đã được nghiên cứu. Theo vị trí của các góc đối với bạc cuống phun, bốn góc được phân loại thành hai nhóm - gần góc và xa góc. Độ lớn của cong vênh của các góc gần lớn hơn so với góc xa cho tất cả các trường hợp được xem xét trong mô phỏng số. Trong giai đoạn điền đầy, các góc gần của phần nhựa được điền đầy nhanh hơn so với các góc xa, do chiều dài dòng chảy ngắn hơn. Do đó, chúng có nhiệt độ nóng chảy thấp hơn và áp lực cao hơn so với các góc xa. Điều này có thể dẫn đến tính lưu động thấp trong giai đoạn nén tiếp theo, vốn được coi là một bước quan trọng để bù cho độ co thể tích bằng cách thêm vật liệu bổ sung. Do đó, sau khi chuyển đổi từ giai đoạn điền đầy đến giai đoạn nén, hầu hết dòng chảy bổ sung gây ra bởi áp lực nén được chuyển tới các góc xa của phần nhựa. Điều này đã được xác minh bằng cách kiểm tra sự thay đổi tốc độ dòng chảy thông qua mỗi miệng phun và giải thích lý do cho việc độ cong vênh cao hơn có thể được tìm thấy tại các góc gần của phần nhựa.

Trong khi áp lực nén cao có xu hướng làm giảm độ cong vênh trong một sản phẩm ép, kết quả mô phỏng trong nghiên cứu này cho thấy độ cong vênh tăng khi tăng áp lực nén. Mặc dù áp lực cao đã được áp dụng trong giai đoạn nén, nó không thể ảnh hưởng một cách hiệu quả tới phẩn nhựa ở các góc gần. Bởi vì độ cong vênh liên quan chặt chẽ đến sự phân bố của độ co rút thể tích, áp lực nến tác động làm giảm sự co rút của phần nhựa ở các góc xa, dẫn đến sự chênh lệch gia tăng giữa độ co ngót giữa các góc gần và xa. Điều này đưa ra một giải thích cho việc độ cong vênh tăng trong trường hợp tăng áp lực nén. Ngoài ra, thời gian điền đầy tỷ lệ nghịch với tốc độ dòng chảy trong giai đoạn điền đầy. Tốc độ dòng chảy thấp tương ứng với thời gian điền đầy dài, do đó dẫn đến sự khác biệt nhỏ hơn trong sự phân bố áp suất gây ra trong giai đoạn điền đầy. Vì sự phân bố áp lực là đồng đều hơn khi tốc độ dòng chảy thấp, nên độ cong vênh của những phần này giảm đi.

\section{c/ Kiểm tra xác thực}

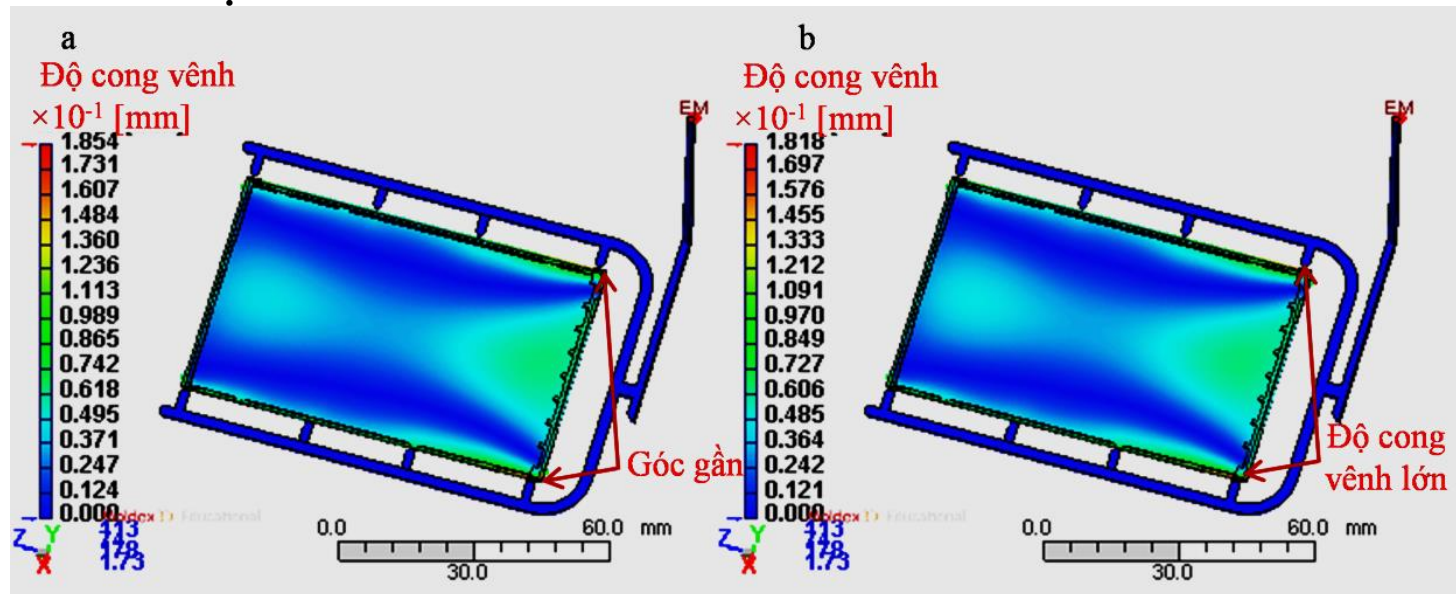

Hình 7: Độ cong vênh thu được bằng mô phỏng số: (a) trong điều kiện cài đặt của trường hợp 4 và (b) trong điều kiện mới từ phân tích tỷ số $\mathrm{S} / \mathrm{N}$. 
Từ phân tích tỷ số $\mathrm{S} / \mathrm{N}$, ta có thể xác định rằng độ cong vênh tối thiểu có thể đạt được từ điều kiện cài đặt A3-B1-C1-D2-E2-F2-G3 như được chỉ ra trong hình 5. Thêm một mô phỏng nữa được thực hiện, dùng những điều kiện này để xác nhận. Kết quả cho thấy rằng độ cong vênh tối đa của sản phẩm là 0,1818 . Giá trị này thấp hơn giá trị độ cong vênh của tất cả các trường hợp được xem xét trong mảng trực giao được liệt kê trong bảng 4 .

Các hình $7 \mathrm{a}$ và $\mathrm{b}$ cho thấy độ cong vênh từ các thông số của trường hợp số 4 trong bảng 4 và trong trường hợp từ phân tích tỷ số $\mathrm{S} / \mathrm{N}$. Từ thử nghiệm xác nhận này, ta có thể kết luận được rằng phương pháp Taguchi có thể tạo ra một sự kết hợp các thông số cài đặt tốt hơn để có thể giảm thiểu cong vênh.

\subsection{Tối ưu hóa để giảm thiểu độ cong vênh}

\section{a/ Tạo mô hình bề mặt đáp ứng}

Phương pháp Taguchi là một công cụ hiệu quả và mạnh mẽ có khả năng phân tích những ý nghĩa tương đối của mỗi thông số cài đặt và đề xuất một bộ thông số kết hợp để giảm thiểu độ cong vênh $[22,23]$. Tuy nhiên, các giá trị tối ưu từ phương pháp Taguchi bị giới hạn ở một trong ba mức được chỉ định cho mỗi thông số. Do đó, RSM đã được sử dụng để giảm độ cong vênh của phần sản phẩm lai hơn nữa. Trong nghiên cứu này, mô hình bề mặt đáp ứng bậc nhất được tạo ra bằng cách sử dụng các kết quả mô phỏng số trong bảng 4. Sử dụng phương pháp bình phương cực tiểu, tất cả các hệ số được xác định, là mô hình đơn giản sau đây. Cần lưu ý rằng các tham số được chuẩn hóa và các ký hiệu được sử dụng để xác định các hệ số.

$$
\begin{array}{r}
\text { Cong vênh }=0.205-6.122 \times 1 \mathrm{O}^{-3} \mathrm{~A}+8.058 \times 1 \mathrm{O}^{-4} \mathrm{~B} \\
+1.24 \mathrm{O} \times 1 \mathrm{O}^{-2} \mathrm{C}+2.658 \times 1 \mathrm{O}^{-3} \mathrm{D}-8.333 \times 1 \mathrm{O}^{-4} \mathrm{E} \\
+1.750 \times 1 \mathrm{O}^{-4} \mathrm{~F}-2.781 \times 1 \mathrm{O}^{-3} \mathrm{G}
\end{array}
$$

Từ mô hình này, thông số $\mathrm{C}$ (áp suất nén) có đóng góp quan trọng nhất vì nó có hệ số lớn nhất. Thông số $\mathrm{A}$ (thời gian điền đầy) với hệ số lớn thứ hai, có đóng góp quan trợng thứ hai. Kết quả này phù hợp với phân tích tỷ số $\mathrm{S} / \mathrm{N}$ trước dựa trên phương pháp Taguchi. Cần lưu ý rằng mô hình bậc nhất cho thấy thông số $\mathrm{G}$ (nhiệt độ ban đầu của phần kim loại chèn) là quan trọng hơn thông số $\mathrm{D}$ (nhiệt độ nóng chảy của nhựa) trong việc giảm thiểu độ cong vênh. Do mức lỗi tương đối lớn $\left(\mathrm{R}^{2}\right.$ là 0.862$)$ trong hồi quy, mô hình cần được cải thiện hơn nữa.

Để cải thiện mô hình RSM, các hệ số bậc hai cho tất cả các tham số được đưa ra trong bước phân tích tiếp theo. Vì mối tương quan giữa các thông số không có có ý nghĩa thống kê, nên các hệ số bậc hai cho tương tác giữa các thông số không được xem xét. Mô hình bề mặt đáp ứng bậc hai cho các thông số đã chuẩn hóa như sau:

$$
\begin{aligned}
& \text { Cong vênh }=0.192-5.777 \times 1 \mathrm{O}^{-3} \mathrm{~A}+3.439 \times 1 \mathrm{O}^{-4} \mathrm{~B} \\
& \quad+8.014 \times 1 \mathrm{O}^{-3} \mathrm{C}+2.658 \times 1 \mathrm{O}^{-3} \mathrm{D}-8.333 \times 1 \mathrm{O}^{-4} \mathrm{E} \\
& \quad+1.75 \mathrm{O} \times 1 \mathrm{O}^{-4} \mathrm{~F}-2.319 \times 1 \mathrm{O}^{-3} \mathrm{G}+1.903 \times 1 \mathrm{O}^{-3} \mathrm{~A}^{2} \\
& -1.111 \times 1 \mathrm{O}^{-3} \mathrm{~B}^{2}-5.707 \times 1 \mathrm{O}^{-5} \mathrm{C}^{2}+5.846 \times 1 \mathrm{O}^{-3} \mathrm{D}^{2} \\
& +4.421 \times 1 \mathrm{O}^{-3} \mathrm{E}^{2}+4.296 \times 1 \mathrm{O}^{-3} \mathrm{~F}^{2}+1.764 \times 1 \mathrm{O}^{-3} \mathrm{G}^{2}
\end{aligned}
$$

Cần lưu ý rằng $\mathrm{R}^{2}$ của các mô hình này có giá trị 0.983 , qua đó chứng minh rằng những mô hình hồi quy phi tuyến này có lỗi chấp nhận được.

Từ các mô hình này, ta có thể xây dựng được các bề mặt đáp ứng để hiển thị các tác động của các thông số cài đặt. Hình 8 cho thấy các ví dụ điển hình của các bề mặt đáp ứng trong nghiên cứu này. Bề mặt đáp ứng mô tả sự tương tác của các thông số $\mathrm{B}$ (thời gian nén) và $\mathrm{E}$ (nhiệt độ khuôn) được thể hiện trong hình $8 \mathrm{a}$. Bề mặt đáp ứng mô tả sự tương tác giữa các thông số $\mathrm{D}$ (nhiệt độ nhựa nóng chảy) và $\mathrm{E}$ (nhiệt độ khuôn) được thể hiện trong hình $8 \mathrm{~b}$. Điểm cực tiểu đại diện cho các giá trị tối ưu cho các thông số tương ứng. 


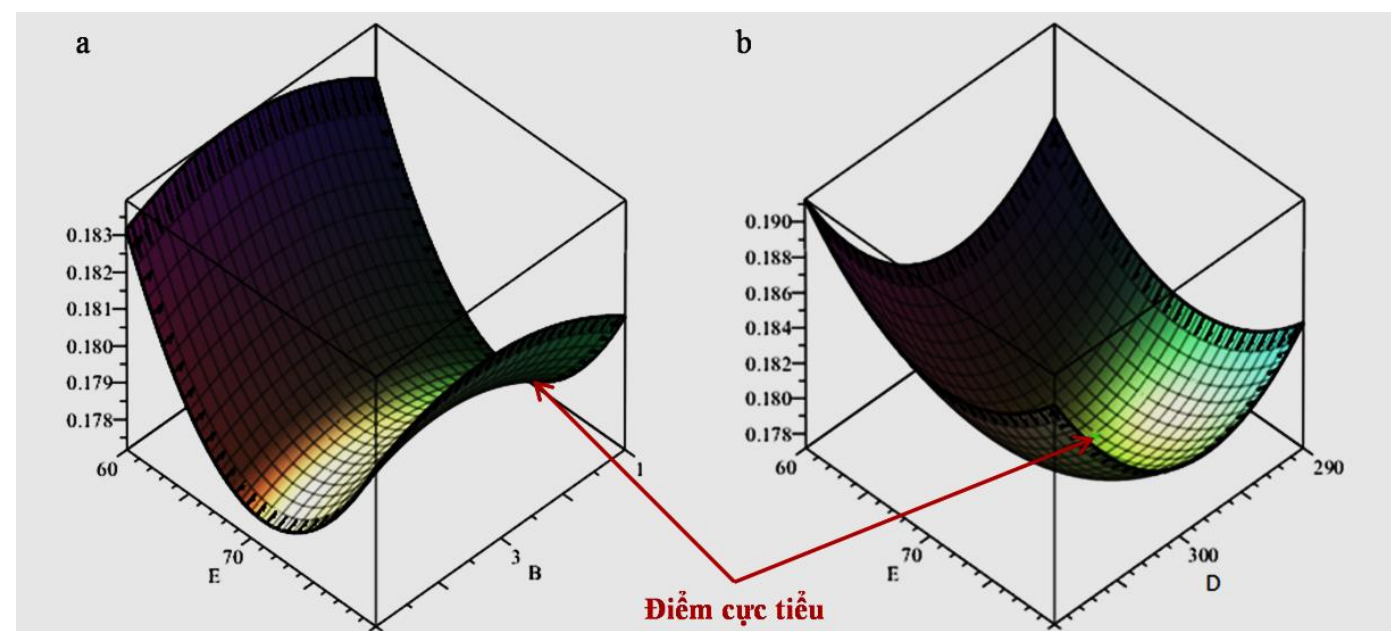

Hình 8: Bề mặt đáp ứng của độ cong vênh đối với hai thông số: (a) $\mathrm{B}$ (thời gian nén) và $\mathrm{E}$ (nhiệt độ khuôn) và (b) $\mathrm{D}$ (nhiệt độ nóng chảy) và $\mathrm{E}$ (nhiệt độ khuôn).

Để xác nhận mô hình hồi quy phi tuyến và mô hình đáp ứng bề mặt được tạo ra, một đồ thị song phân vị Q-Q như trong hình 9 được sử dụng. Bước này không được chú ý bởi các tác giả khác, điều này có thể dẫn đến lãng phí tài nguyên do các mô hình RSM không tối ưu cho công việc của họ. Hình 8 cho thấy mô hình của nghiên cứu này là phân phối chuẩn.

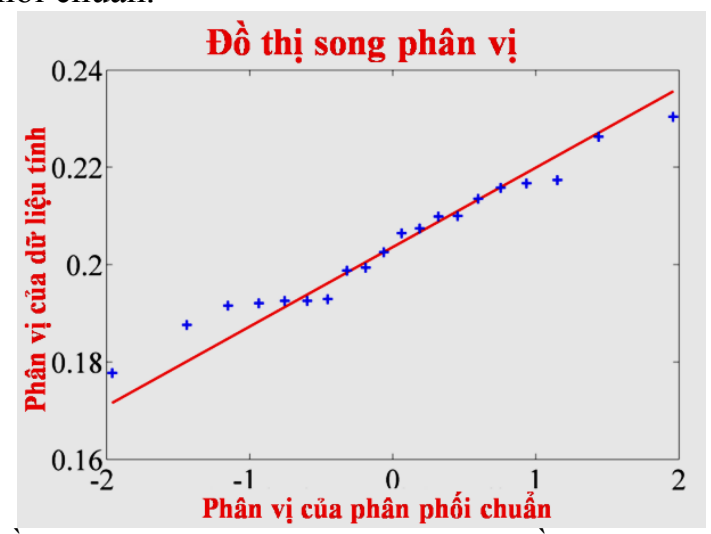

Hình 9: Đồ thị song phân vị Q-Q của mô hình bề mặt đáp ứng hiện tại.

Để xác định các giá trị tối ưu của mỗi thông số cài đặt, qua đó giảm thiểu cong vênh, mô hình bề mặt đáp ứng thu được ở trên đã được sử dụng. Hình 10 trình bày hai đồ thị đại diện của tỷ số $\mathrm{S} / \mathrm{N}$ và độ cong vênh dự đoán từ mô hình bề mặt đáp ứng. Như được chỉ ra trong hình $10 \mathrm{a}$, cả hai phương pháp Taguchi (phân tích tỷ số $\mathrm{S} / \mathrm{N}$ ) và bề mặt đáp ứng (RSM) dự đoán cùng một giá trị tối ưu cho tham số $\mathrm{B}$ (thời gian nén). Tuy nhiên, trong trường hợp tham số $\mathrm{D}$ (nhiệt độ nhựa nóng chảy) trong hình $10 \mathrm{~b}$, phương pháp RSM cung cấp một giá trị chính xác hơn so với phân tích tỷ số $\mathrm{S} / \mathrm{N}$. Như đã đề cập ở trên, lợi thế của phương pháp RSM so với phân tích tỷ lệ $\mathrm{S} / \mathrm{N}$ có thể cho ta những giá trị tối ưu chính xác hơn. Bảng 7 liệt kê các điều kiện tối ưu cho các thông số cài đặt của cả hai phương pháp. Một đoạn code MATLAB ${ }^{\circledR}$ đơn giản được viết và sử dụng để xác định các giá trị tối ưu từ mô hình RSM bậc hai. Mô-đun tối ưu hóa của Maple ${ }^{\circledR}$ cũng cho ra giá trị giống như đoạn code MATLAB ${ }^{\circledR}$, qua đó xác nhận những kết quả này. Điều này cho thấy, ta có thể không cần dùng các thuật toán phức tạp hay các phần mềm thương mại bằng cách sử dụng các công cụ đơn giản, như $M A T L A B \circledR$, để thực hiện nghiên cứu với các tài nguyên tối thiểu. Điều đáng lưu ý là đoạn code hiện tại cũng cho thấy một phương pháp đơn giản để mở rộng các hàm tối ưu hóa cục bộ tích hợp sẵn của MATLAB® ra toàn bộ miền tính toán [24]. 

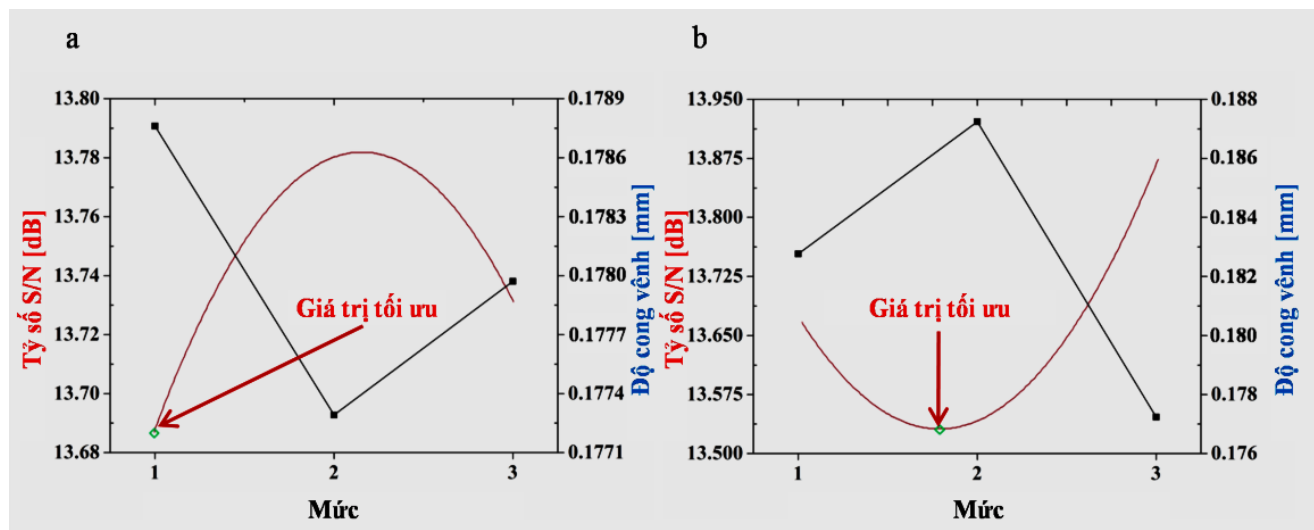

Hình 10: Biểu đồ tỷ số $\mathrm{S} / \mathrm{N}$ và độ cong vênh được ước tính từ mô hình $\mathrm{RSM}$ bậc 2: (a) tác động của thông số $\mathrm{B}$ (thời gian nén) và (b) tác động của thông số $\mathrm{D}$ (nhiệt độ nhựa nóng chảy).

Bảng 7: Các điều kiện cài đặt được đề xuất từ phương pháp Taguchi và mô hình RSM.

\begin{tabular}{|c|c|c|c|}
\hline Ký hiệu & Thông số cài đặt & Taguchi & RSM \\
\hline A & Thời gian điền đầy $(\mathrm{s})$ & 0.5 & 0.5 \\
\hline $\mathrm{B}$ & Thời gian nén $(\mathrm{s})$ & 1 & 1 \\
\hline $\mathrm{C}$ & Áp suất nén $(\mathrm{MPa})$ & 310 & 310 \\
\hline $\mathrm{D}$ & Nhiệt độ nhựa nóng chảy $\left({ }^{\circ} \mathrm{C}\right)$ & 300 & 297.7264 \\
\hline $\mathrm{E}$ & Nhiệt độ khuôn $\left({ }^{\circ} \mathrm{C}\right)$ & 70 & 70.9424 \\
\hline $\mathrm{F}$ & Thời gian làm nguội $(\mathrm{s})$ & 15 & 14.8982 \\
\hline $\mathrm{G}$ & Nhiệt độ ban đầu của phần kim loại chèn $\left({ }^{\circ} \mathrm{C}\right)$ & 35 & 33.2857 \\
\hline \multicolumn{2}{|c|}{ Kết quả cong vênh dự đoán } & N/A & 0.1772 \\
\hline & Kết quả cong vênh xác nhận & 0.1818 & 0.1807 \\
\hline
\end{tabular}

\section{b/ Kiểm tra xác nhận}

Để xác nhận các điều kiện cài đặt tối ưu thu được bằng cách sử dụng RSM, một mô phỏng xác thực được thực hiện. Như được chỉ ra trong bảng 7 , giá trị độ cong vênh tối đa của sản phẩm lai thu được là 0.1807 , nhỏ hơn bất kỳ giá trị độ cong vênh nào khác thu được trong phần trước của nghiên cứu này. Hơn nữa, các góc gần của phần nhựa cho thấy giá trị độ cong vênh lớn hơn so với các góc xa, cũng như trong các trường hợp khác trước đây (như được thể hiện trong hình 11). Bước xác nhận này cho thấy sự kết hợp của kỹ thuật mảng trực giao được sử dụng trong phương pháp Taguchi và RSM, tức là tối ưu hóa kép, cung cấp một cách hiệu quả cho ta các giá trị tối ưu bằng cách sử dụng số lượng tối thiểu thử nghiệm và không yêu cầu thêm các thuật toán, phương pháp tối ưu hóa phức tạp hoặc phần mềm đắt tiền.

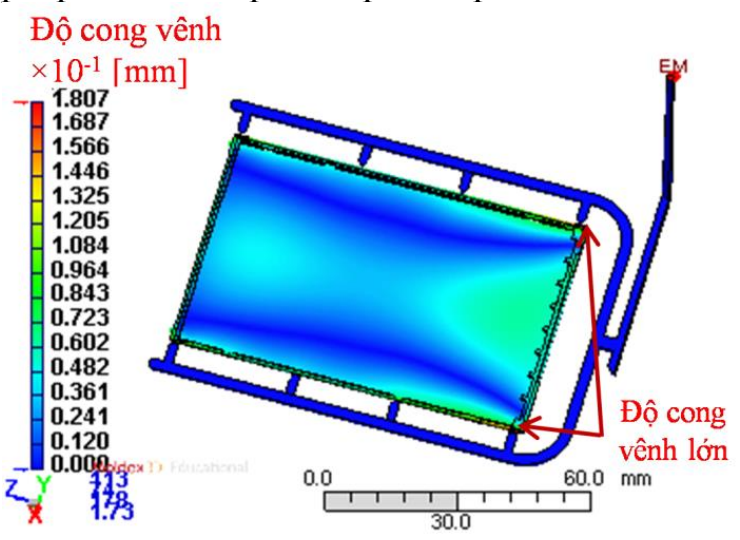

Hình 11: Sự phân bố của độ cong vênh trong điều kiện thông số cài đặt tối ưu.

Cần lưu ý rằng khi bảy thông số được xem xét để tạo ra mô hình bề mặt đáp ứng bậc 2 , thiết kế tổng hợp trung tâm $(\mathrm{CCD})$ và thiết kế Box-Behnken, các kỹ thuật $\mathrm{DOE}$ thường được sử dụng, yêu cầu tương ứng là 79 và 57 thử nghiệm [25]. Khi đó, những thông số cài đặt tối ưu để đạt được độ cong vênh tối thiểu cho sản 
phẩm lai có thể đạt được một cách hiệu quả mà không cần qua bước sàng lọc thông số. Do đó, so với các phương pháp khác, ta có thể xác minh rằng sự kết hợp của phương pháp Taguchi và RSM là đủ để khắc phục những nhược điểm của phương pháp Taguchi như một phương pháp tối ưu hóa rời rạc và cho kết quả tốt hơn trong phạm vi yêu cầu mà không cần sử dụng các thuật toán phức tạp hoặc phần mềm đắt tiền khác.

\section{KẾT LUẬN}

Trong nghiên cứu này, một kỹ thuật tối ưu hóa kép được sử dụng để nghiên cứu ảnh hưởng của các thông số cài đặt lên độ cong vênh của các sản phẩm lai nhựa-kim loại và cũng để xác định các điều kiện tối ưu cho độ cong vênh tối thiểu. Kỹ thuật tối ưu hóa được sử dụng trong nghiên cứu này về cơ bản bao gồm kỹ thuật mảng trực giao của phương pháp Taguchi được sử dụng kết hợp với RSM. Sản phẩm khung tích hợp mới được phát triển đã được chọn làm mô hình nghiên cứu. Trong các mô phỏng số của quá trình IIM của sản phẩm lai, bảy thông số cài đặt được xem xét. Từ phân tích tỷ số $\mathrm{S} / \mathrm{N}$ và $\mathrm{ANOVA}$ dựa trên phương pháp Taguchi với một mảng trực giao $\mathrm{L}_{18}(21 \times 37)$, áp suất nén là thông số quan trọng nhất để giảm thiểu độ cong vênh trong sản phẩm. RSM được áp dụng cho các kết quả phân tích của Taguchi với mô hình hồi quy phi tuyến bậc hai để xác định các điều kiện cài đặt. Từ đó, các giá trị thông số tối ưu được thu thập và xác nhận thông qua mô phỏng số.

Sự đơn giản và hiệu quả của phương pháp tối ưu hóa kép được sử dụng trong nghiên cứu này có thể có ích trong các công nghệ sản xuất khác nhau và các xưởng sản xuất, nơi các công cụ tối ưu hóa thương mại không phải lúc nào cũng có sẵn. Phương pháp này được áp dụng một cách hiệu quả cho quy trình IIM để sản xuất hàng loạt các sản phẩm lai nhựa-kim loại là một ví dụ cụ thể. Các thông số cài đặt khác nhau của quá trình IIM thể hiện tính chất phức tạp vốn có của nó. Do đó, phương pháp hiện tại có thể được sử dụng như một công cụ hiệu quả để sản xuất các sản phẩm lai nhựa-kim loại một cách đáng tin cậy với độ chính xác cao và tiêu thụ nguyên liệu tối thiểu.

\section{LỜI CẢM ƠN}

Công trình nghiên cứu này được thực hiện với sự hướng dẫn và giúp đỡ từ giáo sư Lee Bong-Kee, M3Lab, khoa Cơ Khí, trường đại học quốc gia Chonnam, Hàn Quốc.

\section{TÀI LIỆU THAM KHẢO}

[1] Y.C. Chiang, H.C. Cheng, C.F. Huang, J.L. Lee, Y. Lin and Y.K. Shen, Int. J. Adv. Manuf. Technol., Vol. 55, No. 5-8, pp. 517-526, 2011.

[2] C.-C. Lin, Polym. Eng. Sci., Vol. 52, No. 2, pp. 268-276, 2012.

[3] K. Jin, T.S. Jeong, T.S. Kim, N.S. Kim and B.G. Kim, Int. J. Precis. Eng. Manuf., Vol. 15, No. 12, pp. 2533-2542, 2014.

[4] T.K. Nguyen, C.J. Hwang and B.-K. Lee, International Journal of Precision Engineering and Manufacturing, Vol. 18 (2), pp. 187-195, 2017.

[5] M. La, S.M. Park, W. Kim, C. Lee, C. Kim and D.S. Kim, Int. J. Precis. Eng. Manuf., Vol. 16, No. 8, pp. 1801$1808,2015$.

[6] B. Ozcelik and I. Sonat, Mater. Des., Vol. 30, No. 2, 367-375, 2009.

[7] G.J. Zheng, W. Guo, Q. Wang and X.Y. Guo, J. Mech. Sci. Technol., Vol. 29, No. 10, pp. 4153-4158, 2015.

[8] X. Wang, G. Zhao and G. Wang, Mater. Des., Vol. 47, pp. 779-792, 2013.

[9] M.D. Azaman, S.M. Sapuan, S. Sulaiman, E.S. Zainudin and A. Khalina, Polym. Eng. Sci., Vol. 55, No. 5, pp. 1082-1095, 2015.

[10] B. Zafosnik, U. Bozic and B. Florjanic, Int. J. Precis. Eng. Manuf., Vol. 16, No. 12, pp. 2499-2507, 2015.

[11] P.C. Chen, Y.C. Chen, C.W. Pan and K.M. Li, Int. J. Precis. Eng. Manuf., Vol. 16, No. 4, pp. 647-651, 2015.

[12] H. Kurtaran and T. Erzurumlu, Int. J. Adv. Manuf. Technol., Vol. 27, No. 5-6, pp. 468-472, 2006.

[13] X.P. Li, G.Q. Zhao, Y.J. Guan and M.X. Ma, Mater. Des., Vol. 30, No. 10, pp. 4317-4323, 2009. 

DÙNG PHUOONG PHÁP TAGUCHI VÀ BỀ MẠT ĐÁP ÚNG

[14] H.S. Park and X.P. Dang, Int. J. Precis. Eng. Manuf., Vol. 11, No. 6, pp. 879-890, 2010.

[15] W.L. Chen, C.Y. Huang and C.W. Hung, Eng. Comput., Vol. 27, No. 7-8, pp. 951-966, 2010.

[16] S.C. Nian, C.Y. Wu and M.S. Huang, Int. Commun. Heat Mass, Vol. 61, pp. 102-110, 2015.

[17] W.C. Chen and D. Kurniawan, Int. J. Precis. Eng. Manuf., Vol. 15, No. 8, pp. 1583-1593, 2014.

[18] G. Xu and Z.T. Yang, Int. J. Adv. Manuf. Technol., Vol. 78, No. 1-4, pp. 525-536, 2015.

[19] B. Fan and D. Kazmer, in Proceedings of ANTEC, Nashville, Tennessee, pp 938-942, 2003.

[20] J.M. Park, S.J. Jeong and S.J. Park, Polym. Compos., Vol. 32, No. 8, pp. 1297-1303, 2011.

[21] P.K. Bharti and M.I. Khan, Int. J. of Eng. Sci. and Tech., Vol. 2, No. 9, pp. 4540-4554, 2010.

[22] B.-K. Lee, C.J. Hwang, D.S. Kim and T.H. Kwon, J. Manuf. Sci. Eng., Vol. 130, No. 2, 021010, 2008.

[23] R.H. Myers, D.C. Montgomery and C.M. Anderson-Cook, Response Surface Methodology: Process and Product Optimization Using Designed Experiments, Wiley, New York, 2009, pp. 866-878, 2009.

[24] T.K. Nguyen, C.J. Hwang and B.-K. Lee, in KSPE Annual Spring Conference, Jeju, Korea, May 14-16, pp. 657$658,2014$.

[25] T.K. Nguyen, C.J. Hwang and B.-K. Lee, in International Symposium on Green Manufacturing and Applications 2014, Busan, Korea, June 24-28, pp. P-I-01, 2014.

Ngày nhận bài: 26/11/2018 Ngày chấp nhận đăng: 30/03/2019 